\title{
Report on KOSMOS Summer University at the School of Analytical Sciences Adlershof (Berlin): limits and scales in analytical sciences
}

\author{
M. Montes-Bayón ${ }^{1} \cdot$ J. Kneipp ${ }^{2}$ U. Panne , $^{2,3}$
}

Published online: 10 May 2015

(C) Springer-Verlag Berlin Heidelberg 2015

Analytical problem solving at today's level of complexity can be only understood (taught and learned) using integrative and multidisciplinary approaches. With this vision in mind, the School of Analytical Sciences Adlershof (SALSA) at Humboldt-Universität $\mathrm{zu}$ Berlin has developed a doctorate program funded by the Excellence Initiative of the German Ministry of Education and Research and the German Research Foundation which tries to cover fundamental analytical questions using three main interlinked themes: (1) limits and scales, (2) sensitivity and selectivity, and (3) make and measure. The SALSA fellows and staff organize a summer university every year, addressing the major guiding themes in multidisciplinary discussions with renowned international researchers as invited guests. In 2014, the first summer university received additional funding from Humboldt-Universität's KOSMOS program and was therefore named KOSMOS Summer University. KOSMOS Summer University - celebrated at the HumboldtUniversität's Campus Adlershof between the 16th and 24th of September 2014 - initiated the debate about the first of these guiding themes, limits and scales, as it addresses a very basic aspect of the analytical understanding of nature. The classic analytical term limits identifies the extreme frontiers of analytical observation illustrated by topics such as the detection of single molecules or atoms, chemical imaging on surfaces with

M. Montes-Bayón

montesmaria@uniovi.es

1 Department of Physical and Analytical Chemistry, University of Oviedo, C/Julian Claveria 8, 33006 Oviedo, Spain

2 Department of Chemistry, Humboldt-Universität zu Berlin, Brook-Taylor-Str. 2, 12489 Berlin-Adlershof, Germany

3 BAM Federal Institute for Materials Research and Testing, Richard-Willstaetter-Str. 11, 12489 Berlin, Germany extreme resolution, or observation of ultrafast reactions in real time. The scales theme connects the dynamic range of observations such as comprehensive imaging of structures from the nanometer to millimeter scales or detection of a few molecules simultaneously with analysis of the main components. At the same time, analytical data obtained with different analytical methods through different scales in length, time, and concentration are combined to attain new levels of understanding of an object or analytical sample. Thus, scales and limits are a primary ambition of many scientific disciplines, which may not consider themselves connected to analytical sciences.

In the developed KOSMOS Summer University, the goal was to identify connections between the aspects of limits and scales for potential combinations of optical and other imaging approaches with sensitive and selective mass spectrometry. Different guest lecturers were invited to participate in this event in order to cover different aspects of these topics and to address future directions in which analytical chemists (within interdisciplinary teams) will have to play an important role. Interdisciplinary work in natural sciences is often defined by a common sample or artifact, which requires integration of different methods and disciplines as well as establishing a common language for problem solving. This also holds true for analytical sciences.

Most of the participants in the Summer School were Ph.D. students within the SALSA graduate program together with a number of students coming from the guest lecturers' groups. In total, 44 Ph.D. students from institutions that belong to six different countries (Germany, Switzerland, Spain, Norway, UK, and USA) joined the event.

\section{Teaching analytical sciences}

The KOSMOS Summer University intended to use a different approach for teaching analytical sciences. Analytical sciences 
are often involved in socially controversial and complex science-based technological problems, sometimes "wicked problems". These problems are fact-driven, but open-ended, and have multiple solutions because the data may be inadequate, emotions and risk perception are involved, and ethical and political decisions are at stake. This context requires $s c i-$ entific literacy and cross-curricular competencies with the ability to analyze complex problems from different perspectives and the cognitive flexibility to look for unconventional solutions. In addition to the experiment itself, "scientific inquiry" and the "nature of science" are the most relevant theoretical constructs to describe inquiry-based science education. These constructs connect to the cognitive competence of scientific reasoning and are interwoven with underlying epistemological beliefs. The nature of science concept relates to the fundamental limits of science and the awareness that science is always a social process, which influences the process of scientific inquiry.

Learning by inquiry relates to the intentional process of diagnosing problems, critiquing experiments, distinguishing alternatives, planning investigations, researching conjecture, searching for information, constructing models, debating with peers, and forming coherent arguments. However, with the exception of occasional stories told as historical footnotes to general lectures, case studies are rarely used in teaching of analytical sciences. The heart of our scientific knowledge (i.e., the body of facts, concepts, principles, and paradigms) is traditionally taught through textbook science lectures and, on the surface, demands closed-ended cases with correct answers. Therefore, one of the goals of the 2014 edition of the KOSMOS Summer University was to implement the "learning by inquiry" principle in the enrolled students.

This fact-based teaching no longer corresponds to the experiences and setting of today's students; for digital natives, acquiring facts is no longer obviously in the focus of interest, though acquiring cognitive abilities certainly is. At the heart of SALSA's teaching approach will be students learning through joint, cooperative efforts rather than the teacher conveying his views to the students, which will eradicate the "wisdom can't be told" adage. The learner plays a central role in the learning process, not as a passive recipient of information, but increasingly as an active author, co-creator, evaluator, and critical commentator. Learning is becoming an active process of building meaning for oneself. A hallmark of the inquiry- and case-based studies will be development of the student's higher-order skills for scientific reasoning and decision-making. This will require recognition of the students' aptitudes and attitudes, experience in problem solving, the social learning environment, and scientific and procedural knowledge. Developing an adequate vocabulary, and often a mathematical representation, leads to a cognitive representation and can be linked to prior knowledge and problem solving.

\section{KOSMOS Summer University format}

To achieve the final aim of active and critical learning process, the participating students were randomly separated into six working groups before the Summer School started. Each of these groups was assigned to a specific "analytical challenge" related to the topics covered by the different guest lecturers (within the frame of limits and scales). Publications provided by the guest lecturers were used as entry point for discussion. The scientific topics that were covered are detailed below together with the corresponding guest lecturer.

- "How I can do de novo identification of organoarsenicals in ultra-trace amounts in biota?" Jörg Feldmann, University of Aberdeen, UK

- "The validation and interpretation of multi-block models of several types of measurements in biosciences is an important problem in multivariate analysis." Achim Kohler, Norwegian University of Life Sciences, Aas, Norway

- "Creating directed movement in plant tissues: how to create a coil with specific topology?" Rivka Elbaum, Institute of Plant Sciences and Genetics in Agriculture, Israel

- "Trying to resolve the complexity of biological tissue: limits and scales in mass spectrometry imaging" Andreas Römpp, Justus-Liebig Universität Giessen, Germany

- "How we solved the problem of simultaneous measurement in mass spectrometry?" Gary Hieftje, Indiana University, Bloomington, USA

- "It takes more than high quality spectra: solving medical diagnostic needs with Raman spectroscopy... and business propositions... and thorough problem definitions... and..." Gerwin Puppels, Erasmus Universiteit and River Diagnostics, Rotterdam, the Netherlands

Every group met 3-4 times on average before the Summer School started (e-meetings, because the group members were in different countries) to get familiar with the topic of discussion first (often far from their Ph.D. topics) and then to debate the experiments performed, suggesting alternatives, making research conjectures, and forming coherent arguments on the unclear aspects of the specific challenges. The discussion in small groups facilitated communication and networking possibilities, including the support of each group by a mentor scientist selected from the SALSA faculty. 
Once the Summer School started, the working schedule was established as follows:

Morning sessions: Every day a different group presented their questions/comments on their "analytical challenge" to the corresponding guest lecturer. This was configured in the form of a round table discussion and included a brief introduction on the specific topic. During these sessions, the students gave presentations to other students on topics that were far away from their own Ph.D. work in the presence of the guest lecturer who could clarify specific aspects within the discussion. The advantages observed with this discussion format were

- Participation of external members to the discussion group was active (because they were not intimidated by directly asking the guest lecturer or their colleagues)

- Interaction among participants was increased because they knew each other from the previous e-meetings

- Physical arrangement in the room (with the guest lecturer seated as a panelist in the round table) ensured close contact between the lecturer and the students

Afternoon sessions: Over approximately $1.5 \mathrm{~h}$, the corresponding guest lecturer gave his/her version of the specific challenge covered by the students in the morning session. This was followed by an interactive discussion. This format of preparation and presentation permitted a better understanding of the presentations given by the guest lecturers and also deeper discussions than in regular seminar formats (because some aspects were already covered during the morning sessions).

The specific lectures related to the different analytical challenges were complemented with some interesting contributions regarding, for instance, the evolutionary aspects of analytical sciences, like the lecture given by Freddy Adams from the University of Antwerp, Belgium, who discussed with the students about "The world of yesterday: remembrance of an analytical chemist". Also, the challenge on "Research and teaching: symbiosis or conflict?" given by Gary Hieftje from Indiana University, USA, was highly illustrative for the students who actively participated in the discussions. Lastly, two more challenges were introduced to the audience to be tackled probably in future editions of the Summer University: "Scientific investigation of cultural heritage: an analytical challenge" by Oliver Hahn, BAM Federal Institute for Material Research and Testing, Berlin, Germany and "The challenge of studying iron homeostasis in pathophysiology" by Sophie Vaulont, Institut de Recherche Biomedical, Université Paris Decartes, Paris, France.

\section{Conclusions}

Several important feedback messages were obtained about the KOSMOS Summer University 2014 in the format of the last September edition. Some of them came from the participating students in the form of answers to an anonymous questionnaire given at the end of the meeting.

- The possibility of interacting with the lecturers after having a brief introduction into the specific topic by other colleagues makes the learning process more dynamic and vivid.

- If the lecture shows active interaction with students in a relaxed atmosphere of knowledge exchange, the interest is maintained during the discussing periods $(3 \mathrm{~h}$ with some small breaks) even when the topics are not from the field of personal research of the participating respective student.

- Some of the most mentioned acquired competences (documented by the students) were the ability to grasp the concept of scientific challenge, the discussion with scientists, to provide and accept criticisms, to think "outside the box", a better understanding of physics, spectroscopy, measurements, social behavior, working with colleagues across borders, and to present in an international setting.

- On the idea of future editions, some aspects to be improved were proposed; specifically, to include some practical sessions, a more condensed schedule (e.g., 5-6 days), and to provide some (brief) material to get familiar with all the challenge sessions.

Overall, the KOSMOS Summer University 2014 of limits and scales in analytical sciences was a successful event that helped students in many aspects of their Ph.D. studies. The format connects strongly with the overall mission of SALSA graduate schools: a renewal of analytical science can only be established by connecting analytical thinking to existing mental maps in both education and research. Further editions are being planned.

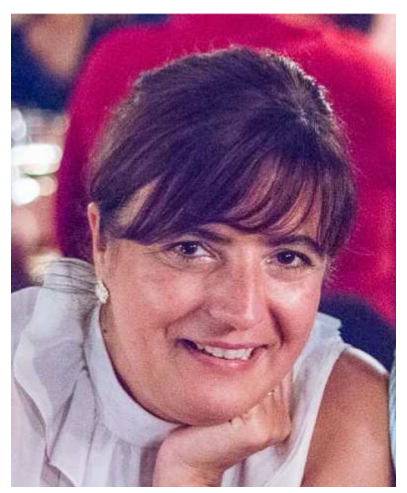

Maria Montes-Bayón KOSMOS Fellow 2014 and SALSA Visiting Professor.montesmaria@uniovi.es. 


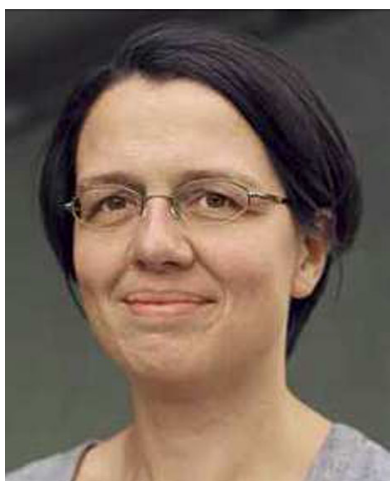

Janina Kneipp KOSMOS Summer University 2014 Organizer.

Janina.kneipp@chemie.huberlin.de.

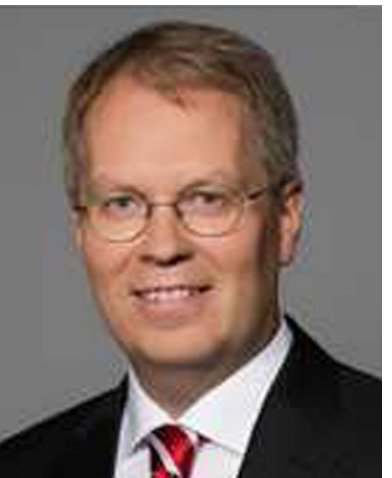

Ulrich Panne KOSMOS Summer University 2014 Organizer.

Ulrich.Panne@BAM.de 